\title{
Hybrid Multicast Transmission for Public Safety Network in 5G
}

\author{
Fei Qi, ${ }^{1}$ Xuetian Zhu, ${ }^{1}$ Xinran Zhang, ${ }^{2}$ and Songlin Sun ${ }^{3}$ \\ ${ }^{1}$ Network Technology \& Planning Department, China Telecom Corporation Limited Beijing Research Institute, Beijing, China \\ ${ }^{2}$ Department of Telecommunication Engineering, Beijing Electronic Science and Technology Institute, Beijing, China \\ ${ }^{3}$ School of Information and Communication Engineering, Beijing University of Posts and Telecommunications, Beijing, China
}

Correspondence should be addressed to Fei Qi; qf13521860822@163.com

Received 28 April 2016; Revised 4 August 2016; Accepted 17 November 2016

Academic Editor: Chenwei Wang

Copyright (C) 2016 Fei Qi et al. This is an open access article distributed under the Creative Commons Attribution License, which permits unrestricted use, distribution, and reproduction in any medium, provided the original work is properly cited.

\begin{abstract}
We investigate the application of wireless multicast technology in public safety network (PSN) in future wireless communication system. The hybrid unicast/multicast transmission system is proposed and analyzed in 3D massive multi-input multioutput (MIMO) channel. The mutual coupling channel model is adopted under the different antenna array configuration scenarios. The proposed hybrid system adopts multicast beamforming in the multicast groups as well as multiuser-MIMO (MU-MIMO) linear precoding in the unicast group to increase system throughput. The null space method based interference cancellation is further performed between each group to eliminate signal leakage generated from each group. Comparisons between two types of antenna array configurations, different channel models, linear precoding as well as multicast beamforming, and user grouping strategies for multicast services are presented and analyzed by simulation.
\end{abstract}

\section{Introduction}

The public safety network (PSN) refers to wireless communication networks used by first responders (FRs) such as police, firefighters, and emergency medical technicians (EMTs) for public protection and disaster relief $[1,2]$. It is an important issue in the design of commercial wireless communication system [3]. Although the network used by FRs is generally private system, the long-term evolution (LTE) system is designed to provide functions in enabling FRs agencies to collaborate with each other as well as provide access service for these agencies when needed. In future fifth-generation (5G) system which features in outstanding physical-layer transmission technologies such as millimeter-wave (mmWave) communication and massive multiple-input multipleoutput (MIMO) transmission [4, 5], the PSN and FR service model has to be reconsidered and reevaluated under the new scenarios. For example, in LTE system and future 5G system, different antenna array configuration schemes in a massive MIMO manner allow the transmitting evolved Node B (eNB) to control both the azimuth and elevation angles of wireless signal propagation and are consequently referred as the threedimensional (3D) MIMO as well [6]. Its flexibility in network deployment and adequate throughput volume advantage will help FRs to facilitate better with cellular network in PSN.

In PSN, the multicast is a promising technology to provide services for FRs $[1,2,7]$. It has been proved to be an efficient and important communication technology and has been standardized in 3rd generation partner project (3GPP) protocols in the form of evolved multimedia broadcast and multicast services (eMBMS). Public Safety Networks (PSNs) have been recognized as the key to a successful response to emergency and disaster situations. In a PSN, first responders often cooperatively accomplish one mission to release as many survivors as possible. Example applications include audio/video conferencing as well as one-to-many data dissemination in critical situations. Multicast is a very efficient way to support group-oriented applications, especially in mobile environments where the spectrum is valuable. This work, correspondingly, addresses that the wireless communication in 5G PSN involves both unicast and multicast traffic and thus formulates a hybrid transmission scenario. In this work we investigate the multicast for PSN in 5G scenario by focusing on the 3D massive MIMO channel model based on the mutual coupling channel model. In the form of physicallayer multicast beamforming, multicast can significantly 
increase system capacity and spectrum efficiency [7-13]. In terms of theoretical analysis, the general wireless multicast beamforming problem is NP-hard problem. In massive MIMO scenario, this problem can be solved in asymptotic analysis with closed-form solutions $[10,11]$, which is important for the design for PSN. Existing literatures have focused on the asymptotic analysis for multicast problem under both perfect channel state information (CSI) scenario and imperfect CSI scenario, which are performed under Rayleigh fading channel model.

To explore the technical insight of multicast in PSN of $5 \mathrm{G}$ scenario, we adopt the 3D MIMO mutual coupling channel model $[14,15]$ to describe the multicast beamforming problem. To proceed, we propose a hybrid transmission system in which the multicast groups adopt multicast beamforming while the unicast group adopts multiuser-MIMO (MU-MIMO) linear precoding. The null space method based interference cancellation between each group is also performed in the proposed system. The optimization of hybrid unicast/multicast precoding is barely investigated and highly challenging. As indicated in [10], even the multicast beamforming problem by itself is already NP-hard, and its optimal solution cannot be obtained efficiently in general. For this reason, this work focuses on the development of a heuristic approach based on null space interference cancellation. We find our proposed hybrid precoding scheme outperforms other heuristic approaches with better efficiency and complexity tradeoff. The system is simulated and analyzed under linear antenna array configuration and rectangular antenna array configuration. We present the comparison amongst different antenna array configurations, channel models, and precoding schemes.

The rest of the paper is organized as follows. Section 2 presents the 3D mutual coupling channel model for the linear antenna array configuration and rectangular antenna array configuration. Section 3 presents the proposed system model. The multicast problem under the mutual coupling channel model and interference cancellation method are modeled and described. The hybrid MU-MIMO linear precoding/multicast beamforming transmission scheme is also described in this section. Section 4 presents the illustrated simulation results, and Section 5 concludes the paper.

Notation. Boldface uppercase and lowercase letters denote matrices and column vectors, respectively. $\mathbb{N}_{+}$and $\mathbb{C}$ denote the set of positive integer and complex number, respectively. $(\cdot)^{*},(\cdot)^{T}$, and $(\cdot)^{H}$ denote complex conjugate, transpose, and Hermitian transpose, respectively. $\mathbf{I}_{N}$ denotes the $N \times N$ identity matrix.

\section{System Architecture}

2.1. Architecture for PSN. Public safety networks (PSNs) support communications for special services including police, fire, and ambulance. In 5G PSN, the requirement has been to develop systems that are highly robust and can address the specific communication needs of emergency services. $5 \mathrm{G}$ system features in providing a massive amount of miscellaneous devices to connect into the packet data network (PDN), the core network. For example, in case of an emergent disaster the operator has to provide the first responders with reliable information as soon as possible. To achieve high network efficiency, 5G PSN will establish a heterogeneous system by incorporating the software defined network (SDN) and radio access network (RAN). Operators may provide multicast services for PSN to efficiently utilize limited bandwidth in the confined RAN coverage area by delivering the identical emergency information for FRs.

For the 5G PSN considering heterogeneity and multicast services, we proposed the PSN architecture as shown in Figure 1. In a future PSN scenario, the heterogeneous PDN in the form of SDN is connected to the RAN of PSN as illustrated. The macrocells are coupled with multiple low power nodes (LPNs) in a ultra-dense manner. The LPNs may apply mmWave communication to provide a much higher speed of data service access to their serving FRs. The FRs within the coverage of the cloud RAN may subscribe to a specific multicast group and utilize multicast beamforming transmission from the heterogeneous access point to receive identical emergency information or subscribe to no multicast groups and utilize MU-MIMO linear precoding to receive highspeed data transmission. In this hybrid service architecture, interference cancellation technology is essential to the system performance [16], since the multicast beamforming transmission and MU-MIMO linear precoding transmission occupy the same time-frequency resources within the coverage of cloud RAN. Consequently, it is crucial to perform the null space method based interference cancellation algorithm to ensure that the manipulated channels of each multicast group as well as the unicast group are orthogonal to each other, resulting in no interference from theoretical point of view.

2.2. Multicast in PSN. One of the most challenging characteristics of PSN is the abrupt intense communication traffic. Although 5G features in providing seamless coverage by densified deployment of LPNs, the traffic peak introduced by PSN can easily paralyse the core network as well as the heterogeneous access points. Also, physical damage to the access points in PSN will contribute to the traffic burden for the whole system. As a result, multicast has been considered as a crucial solution for PSN communication since it can fast spread identical message to the coverage area without introducing further communication costs. In practical system, the multicast can be performed in IP-layer by utilizing various multicast protocols. The IP multicast provides a more efficient method for many-to-many communication and has become more and more important for both commercial networks and dedicated systems. IP multicast allows the source to send a single copy of data, using a single address for the entire group of recipients. The most used multicast routing protocol is the Protocol Independent Multicast Sparse Mode (PIM$\mathrm{SM})$. Routers between the source and recipients use the group address to route the data. The routers forward duplicate data packets only when required; that is, the path to recipients diverges. 


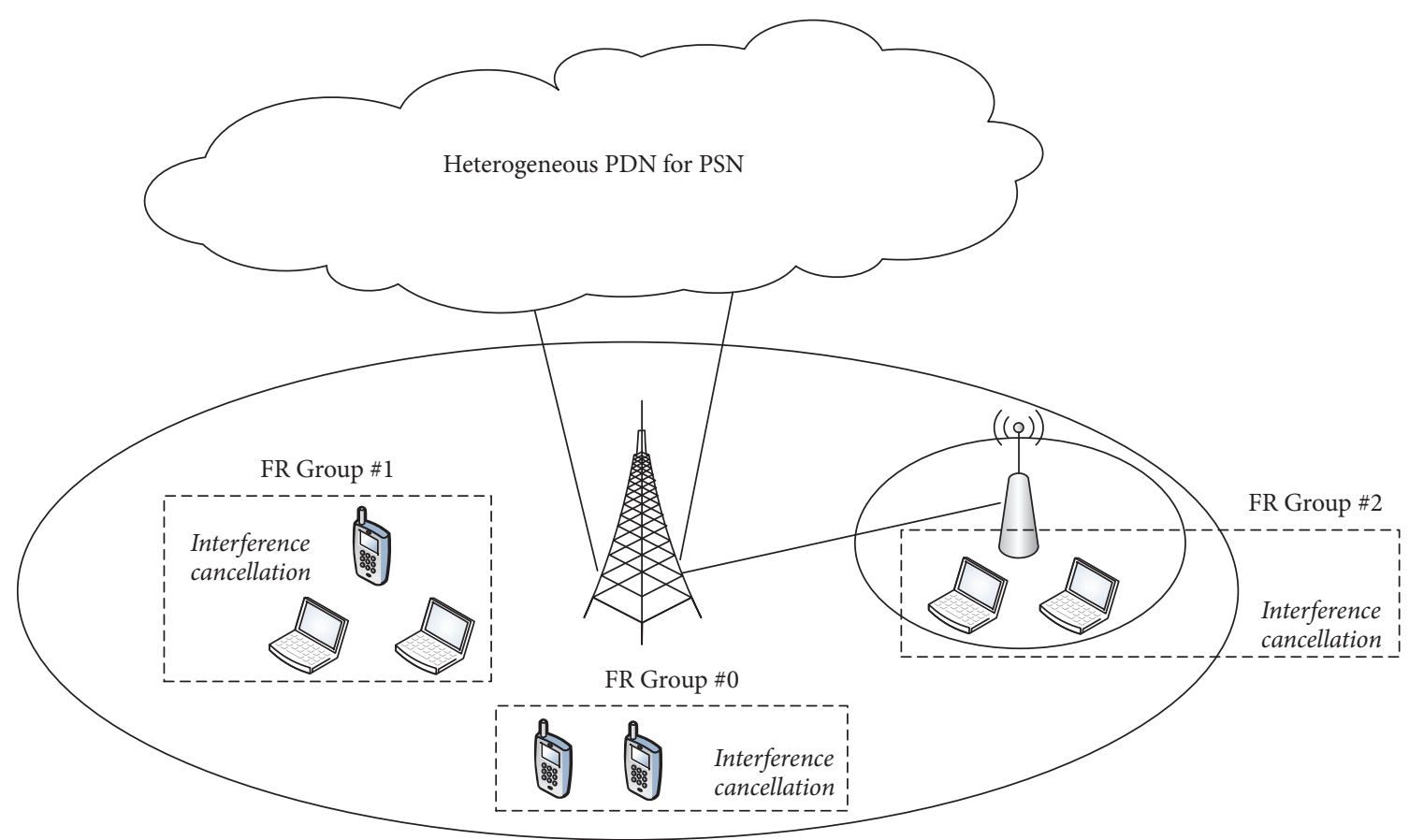

FIgURE 1: Hybrid multicast transmission in PSN.

Although IP-layer multicast has been successfully implemented in practical system, it introduces extra communication complexity for routers and signaling overhead. The wireless communication system, however, has multicast nature in it and is ideal to perform multicast transmission for PSN. In the form of physical-layer wireless multicast, the multicast FRs share the same access medium, that is, wireless spectrum and time; hence it will greatly increase system capacity and lighten the peak traffic problem in PSN. Combined with large-scale antenna array technology in 5G, the multicast is a suitable and effective solution to deliver communication services for FRs. We model the hybrid multicast system for PSN in the following sections.

\section{Channel Model for Multicast in PSN}

In preparation for multicast transmission modeling, we first present the channel modeling work. Considering downlink massive MIMO multicast system where eNB equipped with $N$ antenna broadcasts grouped messages to $K$ FRs (here and after referred to as FRs) equipped with single antenna within eNB's coverage, $N, K \in \mathbb{N}_{+}, N \geq K$. Assuming the nonline-of-sight (NLOS) scenario, the channel model can be described by [6]

$$
\mathbf{G}=\mathbf{H D},
$$

where $\mathbf{G}=\left[\mathbf{g}_{1}, \mathbf{g}_{2}, \ldots, \mathbf{g}_{K}\right]$ denotes the channel matrix and $\mathbf{g}_{k} \in \mathbb{C}^{N \times 1}(k=1,2, \ldots, K)$ denotes the channel vector between eNB and the $k$ th FR. D $=\operatorname{diag}\left\{\sqrt{\beta_{1}}, \sqrt{\beta_{2}}, \ldots, \sqrt{\beta_{K}}\right\}$ denotes the large-scale propagation matrix with $\beta_{k}=\kappa d_{k}^{-\gamma} \xi_{k}$. $\kappa$ is a constant value determined by antenna characteristic and carrier frequency, $d_{k}$ denotes the distance between eNB and the $k$ th FR, $\gamma$ denotes the path loss exponent, and $\xi_{k}$ denotes the log-normal shadow fading factor.

Consider massive MIMO antenna array and 3D MIMO channel model. Since the number of antennas rises to a larger order of magnitude than tradition MIMO antenna array, the mutual impedance of adjacent antennas has to be considered for a more accurate channel model. We adopt the mutual coupling channel model in which $\mathbf{H}=\left[\mathbf{h}_{1}, \mathbf{h}_{2}, \ldots, \mathbf{h}_{K}\right]$ denotes the fast fading matrix, and the column vector $\mathbf{h}_{k} \in \mathbb{C}^{N \times 1}(k=$ $1,2, \ldots, K)$ is described by the following equation $[14,15]$ :

$$
\mathbf{h}_{k}=\mathbf{Z R}_{k} \mathbf{v}_{k} \text {. }
$$

$\mathbf{Z} \in \mathbb{C}^{N \times N}$ denotes the constant mutual coupling matrix related to antenna configuration and has the following form:

$$
\mathbf{Z}=\left(Z_{A}+Z_{L}\right)\left(\boldsymbol{\Gamma}+Z_{L} \mathbf{I}\right)^{-1}
$$

where

$$
\boldsymbol{\Gamma}=\left[\begin{array}{ccccc}
Z_{A} & Z_{M} & 0 & \cdots & 0 \\
Z_{M} & Z_{A} & Z_{M} & \cdots & 0 \\
0 & Z_{M} & Z_{A} & \cdots & 0 \\
\vdots & \vdots & \ddots & \ddots & \vdots \\
0 & 0 & \cdots & Z_{M} & Z_{A}
\end{array}\right] .
$$

The complex value of $Z_{A}, Z_{L}$, and $Z_{M}$ denotes the antenna impedance, load impedance, and mutual impedance, respectively. $\mathbf{R}_{k}$ denotes steering matrix and $\mathbf{v}_{k}$ is the Gaussian stochastic factor. 
The form and size of $\mathbf{R}_{k}$ and $\mathbf{v}_{k}$ depend on antenna array configuration [6]. For linear antenna array scenario, suppose that the $k$ th FR has $A_{k}$ different angles of arrivals (AoAs) denoted by $\theta_{k, l}\left(l=1,2, \ldots, A_{k}\right)$. In that case $\mathbf{R}_{k}$ has $A_{k}$ column of steering vectors; that is, $\mathbf{R}_{k}=\left[\mathbf{r}_{k, 1}, \mathbf{r}_{k, 2}, \ldots, \mathbf{r}_{k, A_{k}}\right]$, where

$$
\begin{gathered}
\mathbf{r}_{k, l}=\mathbf{a}\left(\theta_{k, l}\right)=\frac{1}{A_{k}}\left[1, e^{(j 2 \pi d / \lambda) \sin \theta_{k, l}}, e^{(j 2 \pi d / \lambda) 2 \sin \theta_{k, l}}, \ldots,\right. \\
\left.e^{(j 2 \pi d / \lambda)(N-1) \sin \theta_{k, l}}\right]^{T}
\end{gathered}
$$

$d$ denotes the distance between adjacent antennas, and $\lambda$ denotes the wave length. $\mathbf{a}(\theta)$ is defined as the steering vector function for later convenience.

For the rectangular antenna array scenario, the $k$ th FR has $A_{k}$ different azimuths of arrivals and elevations of arrivals (EoAs) denoted by $\theta_{k, i}$ and $\phi_{k, i}$, respectively. In that case the column steering vectors in $\mathbf{R}_{k}=\left[\mathbf{r}_{k, 1}, \mathbf{r}_{k, 2}, \ldots, \mathbf{r}_{k, A_{k}}\right]$ is described by the following equation:

$$
\begin{aligned}
\mathbf{r}_{k, l} & =\frac{1}{A_{k}} \times \operatorname{vec}\left[\mathbf{a}\left(\theta_{k, l}\right) \otimes \mathbf{a}\left(\phi_{k, l}\right)^{T}\right]=\frac{1}{A_{k}} \times \operatorname{vec}\{[1, \\
& \left.e^{(j 2 \pi d / \lambda) \sin \theta_{k, l}}, e^{(j 2 \pi d / \lambda) 2 \sin \theta_{k, l}}, \ldots, e^{(j 2 \pi d / \lambda)(N-1) \sin \theta_{k, l}}\right] \\
& \otimes\left[1, e^{(j 2 \pi d / \lambda) \sin \phi_{k, l}}, e^{(j 2 \pi d / \lambda) 2 \sin \phi_{k, l}}, \ldots,\right. \\
& \left.\left.e^{(j 2 \pi d / \lambda)(N-1) \sin \phi_{k, l}}\right]\right\},
\end{aligned}
$$

where the symbol $\otimes$ represents the Kronecker product of matrices, and the $\operatorname{vec}(\cdot)$ function denotes vectorization of matrix.

With the established channel model above, the transmission and service model can be accurately described. In the following section we present the proposed hybrid transmission system for multicast services.

\section{Hybrid Multicast Transmission for PSN}

4.1. FR Grouping. Consider $M$ different multicast groups indexed as $1,2, \ldots, M$, and define the group indexed with 0 as the unicast group for nonmulticast FRs. Each user can subscribe to no more than one specific multicast group to receive multicasted information. To characterize that, define the subscription vector $\mathbf{m}=\left\{m_{1}, m_{2}, \ldots, m_{K}\right\}$, where the element $m_{k} \in\{0,1,2, \ldots, M\}(k=1,2, \ldots, K)$ represents that the $k$ th user is subscribed to multicast group indexed with $m_{k}$ (for unicast FRs, $\left.m_{k}=0\right)$. For the grouping method, define $\mathbf{g}_{m}=$ $\left\{g_{1}, g_{2}, \ldots, g_{K}\right\}$ for group $m(m=0,1,2, \ldots, M)$, where $g_{k} \in$ $\{0,1\}(k=1,2, \ldots, K)$ denotes grouping scheme with $g_{k}=1$ being the $k$ th user subscribed to group $m$ and $g_{k}=0$ being otherwise. For FRs in group $m$, let $\mathscr{K}_{m}=\left\{k \mid m_{k}=m\right\}$ and let $\mathscr{K}_{m} \in \mathbb{N}_{+}$denote the set of indices of FRs in group $m$. Let $K_{m}$ denote the number of FRs in group $m$ with $\sum_{m=0}^{M} K_{m}=K$.
4.2. Interference Cancellation. Define channel grouping matrix $\mathbf{G}_{m}=\left[\mathbf{g}_{m, 1}, \mathbf{g}_{m, 2}, \ldots, \mathbf{g}_{m, K_{m}}\right]$, where vector $\mathbf{g}_{m, j} \in$ $\mathbb{C}^{N \times 1}\left(j=1,2, \ldots, K_{m}\right)$ denotes the channel vector for the $j$ th user in group $m$. Before describing the interference cancellation, the receiving signal model is addressed in (7) firstly for the full view of the whole system.

$$
\begin{aligned}
\mathbf{Y} & =\sqrt{P} \mathbf{G} \mathbf{J} \mathbf{S}+\mathbf{z}, \\
\mathbf{z} & =\left[\mathbf{z}_{0}, \mathbf{z}_{1}, \ldots, \mathbf{z}_{M}\right]^{T}, \\
\mathbf{S} & =\left[\mathbf{s}_{0}, \mathbf{s}_{1}, \ldots, \mathbf{s}_{M}\right]^{T}, \\
\boldsymbol{\omega} & =\operatorname{diag}\left(\mathbf{\Omega}_{0}, \mathbf{w}_{1}, \ldots, \mathbf{w}_{M}\right), \\
\mathbf{J} & =\left[\mathbf{J}_{0}, \mathbf{J}_{1}, \ldots, \mathbf{J}_{M}\right], \\
\mathbf{G} & =\left[\mathbf{G}_{0}, \mathbf{G}_{1}, \ldots, \mathbf{G}_{M}\right]^{T}, \\
\mathbf{Y} & =\left[\mathbf{y}_{0}, \mathbf{y}_{1}, \ldots, \mathbf{y}_{M}\right]^{T},
\end{aligned}
$$

where $P$ is the signal power in the transmitter and $\mathrm{Y}$ denotes the receiving signal of the transmitted signal $\mathbf{S}$, which has been processed by the projection matrix $\mathbf{J}$ and precoding matrix $\boldsymbol{\omega}$. The white Gaussian noise is denoted as z. For the matrix $\mathbf{w}_{m}$, it can be described as $\mathbf{w}_{m}=(1 / \lambda)\left[\widehat{\mathbf{g}}_{m, 1}^{*}\right.$, $\left.\widehat{\mathbf{g}}_{m, 2}^{*}, \ldots, \widehat{\mathbf{g}}_{m, K_{m}}^{*}\right]$, in which $\lambda$ is the normalization factor, which can make sure $\boldsymbol{\omega}_{m}=(1 / \lambda) \sum_{j=1}^{K_{m}} \widehat{\mathbf{g}}_{m, 1}^{*}$ and $\boldsymbol{\Omega}_{0}$ is the precoding matrix of the unicast users.

Considering downlink multicast service transmission, in order to cancel the interferences between all groups, we perform the null space method based interference cancellation algorithm [17] in the system. In order to cancel the interferences between all $M$ multicast groups as well as the unicast group, the projection matrix for the channel matrix of group $m$ must satisfy that it spans a subspace of the null space of the channel vectors of underlying victim FRs in all other $M$ groups. For group $m$, the joint channel matrix for FRs in all other $M$ groups is defined as follows:

$$
\mathbf{G}_{\bar{m}}=\left[\mathbf{G}_{0}, \mathbf{G}_{1}, \ldots, \mathbf{G}_{m-1}, \mathbf{G}_{m+1}, \ldots, \mathbf{G}_{M}\right]
$$

By singular value decomposition (SVD), the projection matrix for group $m$ denoted by $\mathbf{J}_{m} \in \mathbb{C}^{N \times N}$ is derived by the following equations:

$$
\begin{aligned}
\mathbf{G}_{\bar{m}} & =\mathbf{U}_{\bar{m}} \boldsymbol{\Lambda}_{\bar{m}} \mathbf{V}_{\bar{m}}, \\
\mathbf{J}_{m} & =\mathbf{V}_{\bar{m}}^{0}\left(\mathbf{V}_{\bar{m}}^{0}\right)^{H}, \\
\mathbf{U}_{\bar{m}} & \in \mathbb{C}^{N \times N}, \\
\mathbf{V}_{\bar{m}} & \in \mathbb{C}^{\left(K-K_{m}\right) \times\left(K-K_{m}\right)}, \\
\boldsymbol{\Lambda}_{\bar{m}} & \in \mathbb{C}^{N \times\left(K-K_{m}\right)} .
\end{aligned}
$$

$\mathbf{U}_{\bar{m}}, \mathbf{V}_{\bar{m}}$, and $\Lambda_{\bar{m}}$ are SVD factor matrices. $\mathbf{V}_{\bar{m}}^{0} \in \mathbb{C}^{\left(K-K_{m}\right) \times N}$ is a subspace of column vector space of $\mathbf{V}_{\bar{m}}$. By multiplying $\mathbf{J}_{m}$, 
the new channel matrix for group $m$ denoted as $\widehat{\mathbf{G}}_{m}$ and new channel vector for the $j$ th user in group $m$ denoted as $\widehat{\mathbf{g}}_{m, j}$ are transformed as follows:

$$
\begin{aligned}
\widehat{\mathbf{G}}_{m} & =\mathbf{J}_{m} \mathbf{G}_{m}, \\
\widehat{\mathbf{g}}_{m, j} & =\mathbf{J}_{m} \mathbf{g}_{m, j} .
\end{aligned}
$$

After the manipulation, the signal leakage from each group can be eliminated, guaranteeing the performance of the hybrid multicast/unicast transmission. In the following parts we will adopt the new channel matrices for analysis.

4.3. Formulation of FR Multicast. In this part we model the multicast problem based on previous channel modeling work. For simplicity, consider local cell multicast scenario only. Let $\boldsymbol{\omega}_{m} \in \mathbb{C}^{N \times 1}$ be the multicast beamforming vector for multicast group $m$ with $\left\|\boldsymbol{\omega}_{m}\right\|^{2}=1$. Let $s_{m}$ be the stochastic information with unit power multicasted in group $m$. Then the transmitted signal in group $m$ is given by

$$
\mathbf{x}_{m}=\sqrt{p_{m}} \boldsymbol{\omega}_{m} s_{m}
$$

where $p_{m}$ is the transmission power for group $m$. Assume that all groups share the same spectrum, then the received signal for the $j$ th user in group $m$ is given by

$$
\begin{aligned}
y_{m, j} & =\widehat{\mathbf{g}}_{m, j}^{H} \mathbf{x}_{m}+\sum_{n \neq m}^{M} \widehat{\mathbf{g}}_{m, j}^{H} \mathbf{x}_{n}+z_{m, j} \\
& =\sqrt{p_{m}} \widehat{\mathbf{g}}_{m, j}^{H} \boldsymbol{\omega}_{m} s_{m}+\sum_{n \neq m}^{M} \sqrt{p_{n}} \widehat{\mathbf{g}}_{m, j}^{H} \boldsymbol{\omega}_{n} s_{n}+z_{m, j},
\end{aligned}
$$

where $z_{m, j}$ is the additive Gaussian noise with zero mean and variance being $\sigma^{2}$. The unperfect precoding scheme causes the interference from intragroup to be the main interference as intergroup interference would be 0 after proper precoding. Then the received SINR for the $j$ th user in group $m$ is

$$
\operatorname{SINR}_{m, j}=\frac{p_{m}\left|\widehat{\mathbf{g}}_{m, j}^{H} \boldsymbol{\omega}_{m} s_{m}\right|^{2}}{\sum_{n \neq m}^{M} p_{n}\left|\widehat{\mathbf{g}}_{m, j}^{H} \boldsymbol{\omega}_{n} s_{n}\right|^{2}+\sigma^{2}} .
$$

In massive MIMO scenario, it is assumed that transmission power per antenna scales inversely to the number of antennas [5]; that is, $p_{m}=\rho_{m}(E / N)$, where $E$ denotes the total transmission power of the base station and $\rho_{m} \in[0,1]$ denotes the power ratio of group $m$. Consider the perfect CSI scenario for simplicity; the multicast problem is formulated as the max-min fairness (MMF) problem [10] as follows:

$$
\begin{aligned}
\mathscr{P}: \max _{\left\{\boldsymbol{\omega}_{m}, \rho_{m}, \mathbf{m}\right\}} \min _{0 \leqslant m \leqslant M} & \min _{\forall j \in \mathscr{K}_{m}} \frac{\rho_{m}(E / N)\left|\widehat{\mathbf{g}}_{m, j}^{H} \boldsymbol{\omega}_{m} s_{m}\right|^{2}}{\sum_{n \neq m}^{M} \rho_{n}(E / N)\left|\widehat{\mathbf{g}}_{m, j}^{H} \boldsymbol{\omega}_{n} s_{n}\right|^{2}+\sigma^{2}} \\
\text { s.t. } \quad & \left\|\boldsymbol{\omega}_{m}\right\|^{2}=1, \quad \forall m \\
& \sum_{m=0}^{M} \rho_{m} \leqslant 1, \quad \rho_{m} \in[0,1] \\
& \mathbf{m}=\left[m_{1}, m_{2}, \ldots, m_{K}\right], \quad m_{k} \in\{0,1, \ldots, M\}, k \in\{1,2, \ldots, K\} \\
& \mathscr{K}_{m}=\left\{k \mid m_{k}=m\right\} .
\end{aligned}
$$

4.4. Asymptotic Optimal Multicast Beamforming. The multicast problem $\mathscr{P}$ can be solved under massive MIMO scenario by asymptotic solution. Similar to existing literatures $[10,11]$, we readily have the following theorem.

Theorem 1. The asymptotic optimal beamforming vector $\boldsymbol{\omega}_{m}^{*}$, optimal power ratio $\rho_{m}^{*}$, and asymptotic minimum SINR achieved in group $m$ are given by

$$
\begin{aligned}
& \boldsymbol{\omega}_{m}^{*}=\alpha_{m} \sum_{j \in \mathscr{K}_{m}} \frac{\widehat{\mathbf{g}}_{m, j}^{H}}{\beta_{j}}, \quad \forall m, \\
& \alpha_{m}=\left(N \sum_{j \in \mathscr{K}_{m}} \beta_{j}^{-1}\right)^{-1 / 2},
\end{aligned}
$$

$$
\begin{aligned}
\rho_{m}^{*} & =\frac{\sum_{j \in \mathscr{K}_{m}} \beta_{j}^{-1}}{\sum_{k=1}^{K} \beta_{k}^{-1}}, \quad \forall m, \\
\operatorname{SINR}_{m}^{\infty} & =\frac{E}{\sigma^{2} \sum_{k=1}^{K} \beta_{k}^{-1}}, \quad \forall m .
\end{aligned}
$$

The theorem indicates that when $N \rightarrow \infty$, the SINR achieved in each group is irrelevant to the user grouping scheme and only depends on large-scale channel attenuation and total number of FRs in the macrocell. In practical massive MIMO scenario the antenna number is large enough to observe the asymptotic behavior above. Existing literatures also indicate that this phenomenon can be affected by pilot contamination in a time-division duplexing (TDD) system. Unlike previous works that focus on the analysis of problem (14a), (14b), (14c), (14d), and (14e) in different scenarios, we 
focus on the hybrid transmission scheme in practical massive MIMO system and directly adopt the asymptotic results from Theorem 1 as the multicast beamformer and power ratio for the simulation system.

4.5. Hybrid Multicast Transmission. With the above multicast model, we investigate several types of MU-MIMO linear precoding schemes $[17,18]$ for the unicast group that is widely adopted in MIMO system for comparison. For the $K_{0} \times 1$ multiuser transmit vector, the precoding matrix $\Omega_{0} \in \mathbb{C}^{N \times K_{0}}$ is multiplied to the transmit vector to perform MU-MIMO linear precoding as follows.

MRT Precoding. The maximum ratio transmission (MRT) precoding algorithm constructs a matched filter in spatial domain. The MRT precoding matrix for the unicast group is denoted as follows:

$$
\begin{aligned}
\boldsymbol{\Omega}_{0, \mathrm{MRT}} & =\frac{1}{\beta_{0}} \widehat{\mathbf{G}}_{0}^{*}, \\
\beta_{0} & =\sqrt{\frac{\operatorname{tr}\left(\mathbf{B}_{0} \mathbf{B}_{0}^{H}\right)}{p_{0}}}, \\
\mathbf{B}_{m} & =\mathbf{G}_{0}^{*} .
\end{aligned}
$$

$B D$ Precoding. The block diagonalization (BD) precoding algorithm is derived with the same manner as the null space method based interference cancellation method from (9) and (10), where the precoding matrix $\boldsymbol{\Omega}_{0, \mathrm{BD}}$ guarantees that the precoded channel matrix of a specific FR lies within the null space of the joint channel matrix of other FRs in the same group; hence interferences from all other FRs are eliminated. The mathematical notations are similar to (9) and (10) and are omitted for simplicity.

These MU-MIMO linear precoding schemes are performed in the unicast group while the multicast beamforming scheme from Theorem 1 is performed in the multicast groups. The proposed hybrid system can provide high-speed transmission for unicast FRs and simultaneously provide multicast services to multicast FRs, increasing the spectrum efficiency of the system greatly.

\section{Simulation Results}

We evaluate the performance of the proposed hybrid system using MATLAB simulation. We focus on the channel model, antenna array configuration, and precoding/beamforming comparison as our major concerns in the simulation system. The linear antenna array and rectangular antenna array are chosen for analysis. Simulations of MRT/BD precoding as well as the multicast beamforming are run with and without the mutual coupling channel model for comparison. For all the multicast service groups, the illustrated performance metrics are calculated as the average metrics. The beamformer is calculated by Theorem 1, while the two MU-MIMO linear precoding schemes are performed in the unicast group. The major parameters of the simulation system are listed in Table 1 .
TABLE 1: Simulation parameters.

\begin{tabular}{ll}
\hline Parameters & Values \\
\hline Transmit antenna number & $N=64$ \\
FR number & $K=16 / 32$ \\
Antenna interval for linear & 0.5 wavelengths \\
antenna array & 0.5 wavelengths for \\
Antenna interval for & horizontal and \\
rectangular antenna array & vertical direction \\
$Z_{A}$ & $50 \Omega$ \\
$Z_{L}$ & $50 \Omega$ \\
$Z_{M}$ & $50 \Omega$ \\
SNR & $-10 \mathrm{~dB}$ to $25 \mathrm{~dB}$ \\
Frequency & $38 \mathrm{GHz}$ \\
Tx antenna height & $36 \mathrm{~m}$ \\
Rx antenna height & $1.5 \mathrm{~m}$ \\
Tx Rx antenna gain & $25 / 13.3$ \\
Scenario & NLOS \\
\hline
\end{tabular}

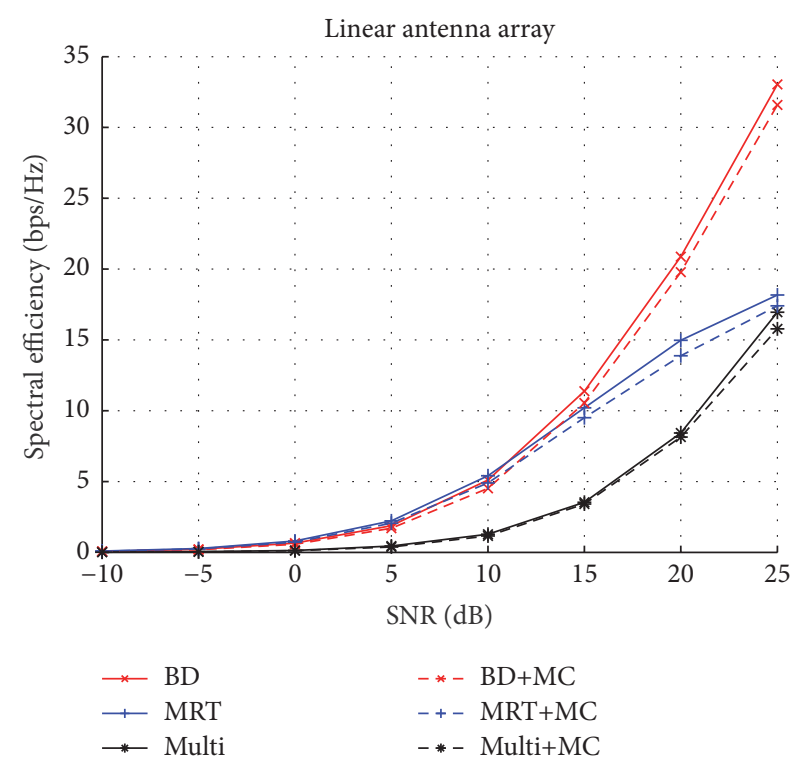

FIgURE 2: Spectrum efficiency (linear antenna array; number of UEs: 16; +MC: mutual coupling; precoding schemes: BD/MRT/ multicast).

5.1. Analysis for the Mutual Coupling Effect. Figures 2 and 3 illustrate the spectrum efficiency of linear antenna array and rectangular antenna array, respectively. The performance of MRT/BD precoding (here and after denoted as MRT and $\mathrm{BD}$ in the legends) and multicast beamforming (here and after denoted as multi in the legends) are evaluated with and without mutual coupling (here and after denoted as MC in the legends) based channel model. Since the mutual coupling effect introduces extra correlation between adjacent antennas, the channel becomes less independent in a spatial prospect hence the performance of all precoding and 


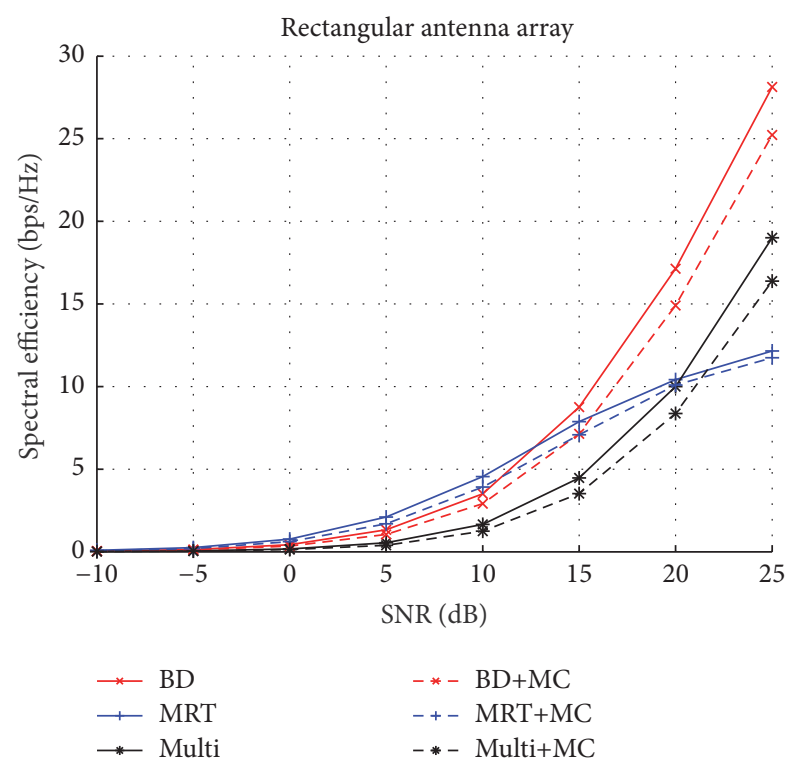

FIGURE 3: Spectrum efficiency (rectangular antenna array; number of UEs: 16; +MC: mutual coupling; precoding schemes: BD/MRT/ multicast).

beamforming schemes are expected to be degraded. This is clearly observed in Figures 2 and 3 as the spectrum efficiency degrades for all precoding and beamforming schemes for both antenna array configurations. Also, since the mutual coupling effect of rectangular antenna array is considered to be more prominent than the linear antenna array, the performance degradation of rectangular antenna array is worse compared to linear antenna array as observed.

\subsection{Comparison between Linear Precoding and Multicast} Beamforming. To compare the linear precoding with multicast beamforming, we first choose the $\mathrm{BD}$ precoding for unicast FRs. Figures 4 and 5 illustrate the spectrum efficiency comparison between $\mathrm{BD}$ precoding and multicast beamforming for linear antenna array and rectangular antenna array, respectively. The total spectrum efficiency for the entire system is illustrated and becomes legend as "all group" for observation convenience. For linear antenna array, the performance of multicast beamforming is worse than $\mathrm{BD}$ precoding. For rectangular antenna array, however, despite the fact that the performance of $\mathrm{BD}$ precoding and multicast beamforming both degrade, the multicast beamforming suffers much less degradation than BD precoding. Similar phenomena are observed in Figures 6 and 7 which illustrate the comparison between MRT precoding and multicast beamforming. This is because the multicasted information for each beamforming user is the same. With a higher correlation between antennas in rectangular antenna array, the signal leakage of multicast beamforming between each user is more of in-phase component and contributes less to the interference for the intended multicast user than that of the unicast group. Hence the multicast beamforming has better performance than linear precoding in terms of channel degradation introduced by concentrated antenna array configuration.

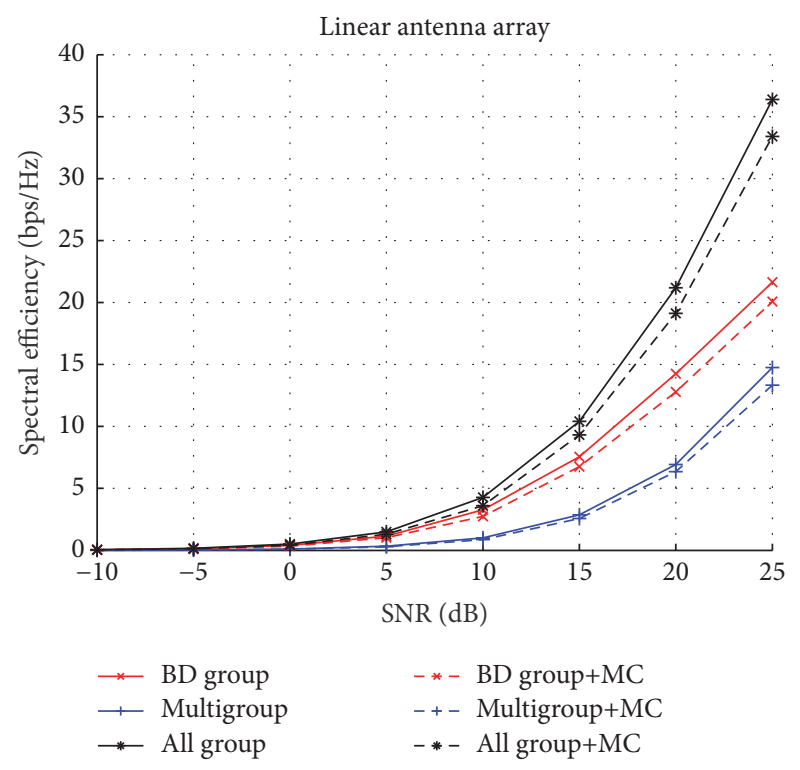

FIGURE 4: Comparison between BD precoding and multicast beamforming (linear antenna array; number of unicast UEs: 8; number of multicast group UEs: 8; intergroup interference cancellation: null space; +MC: mutual coupling; unicast precoding Scheme: BD; multicast precoding scheme: multicast precoding).

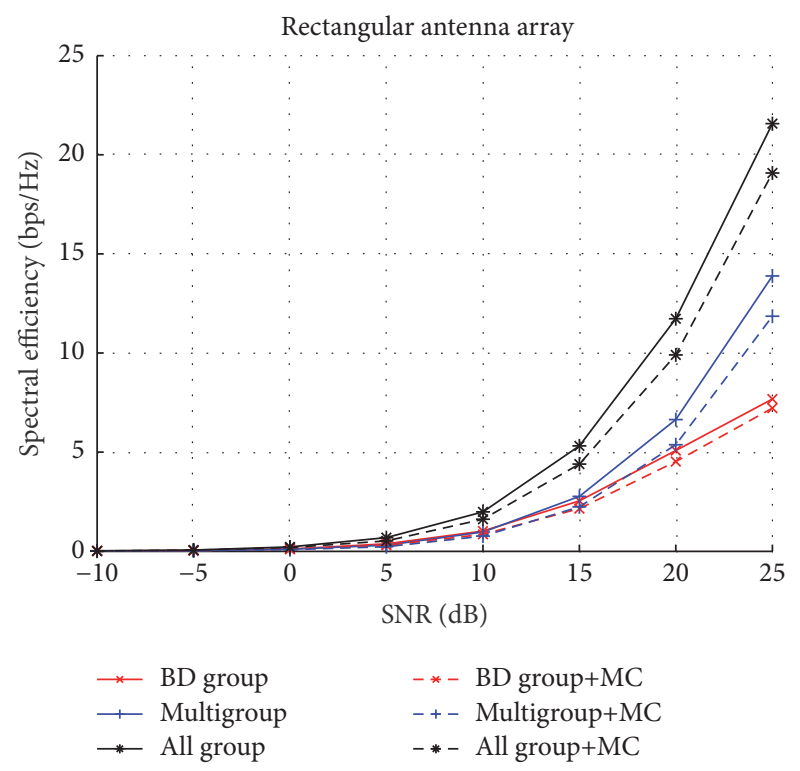

FIGURE 5: Comparison between BD precoding and multicast beamforming (rectangular antenna array; number of unicast UEs: 8; number of multicast group UEs: 8; intergroup interference cancellation: null space; +MC: mutual coupling; unicast precoding scheme: $\mathrm{BD}$; multicast precoding scheme: multicast precoding).

5.3. Analysis for the Grouping Strategy. Figures 8, 9, and 10 illustrate the trend of total spectrum efficiency with an increasing multicast user number while total user number is fixed to be 32. Specifically, a scenario with a much higher correlation between adjacent antennas for rectangular antenna array is presented in Figure 10. With an increasing number of 


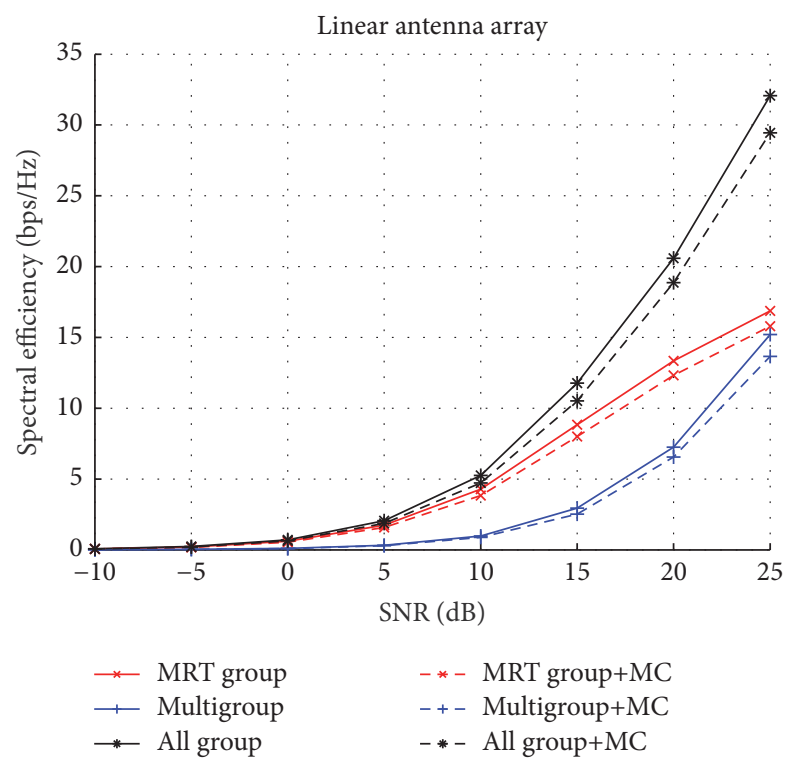

Figure 6: Comparison between MRT precoding and multicast beamforming (linear antenna array; number of unicast UEs: 8; number of multicast group UEs: 8; intergroup interference cancellation: null space; +MC: mutual coupling; unicast precoding scheme: MRT; multicast precoding scheme: multicast precoding).

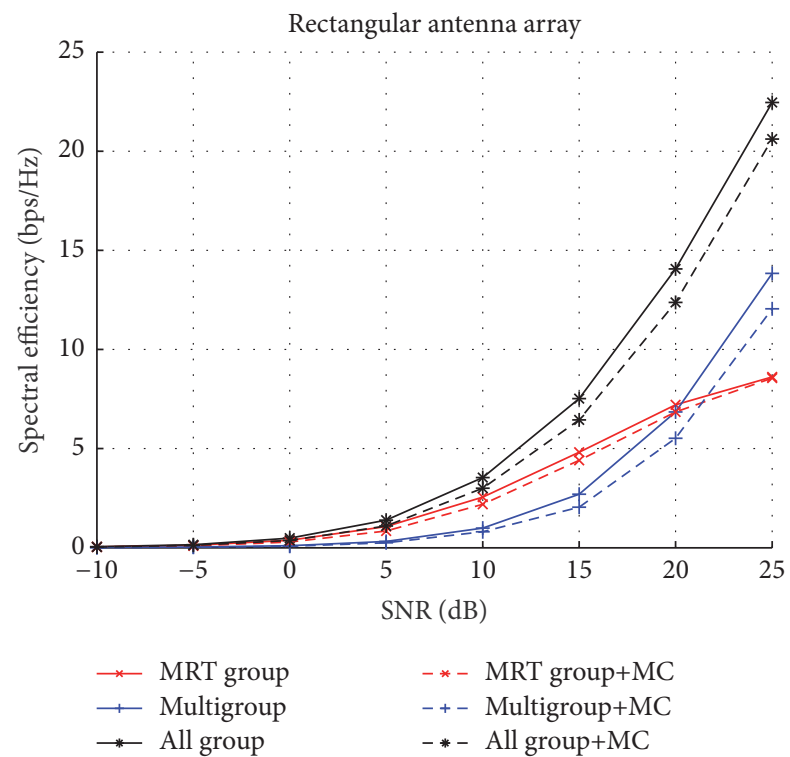

FIGURE 7: Comparison between MRT precoding and multicast beamforming (rectangular antenna array; number of unicast UEs: 8; number of multicast group UEs: 8; intergroup interference cancellation: null space; +MC: mutual coupling; unicast precoding scheme: MRT; multicast precoding scheme: multicast precoding).

multicast FRs, the total spectrum efficiency decreases since the multicast beamforming achieves worse performances than linear precoding in low correlation scenario. Meanwhile in Figure 10 we observe that, in high correlation scenario, the multicast beamforming outperforms linear precoding greatly and hence contributes to the growing trend of total spectrum

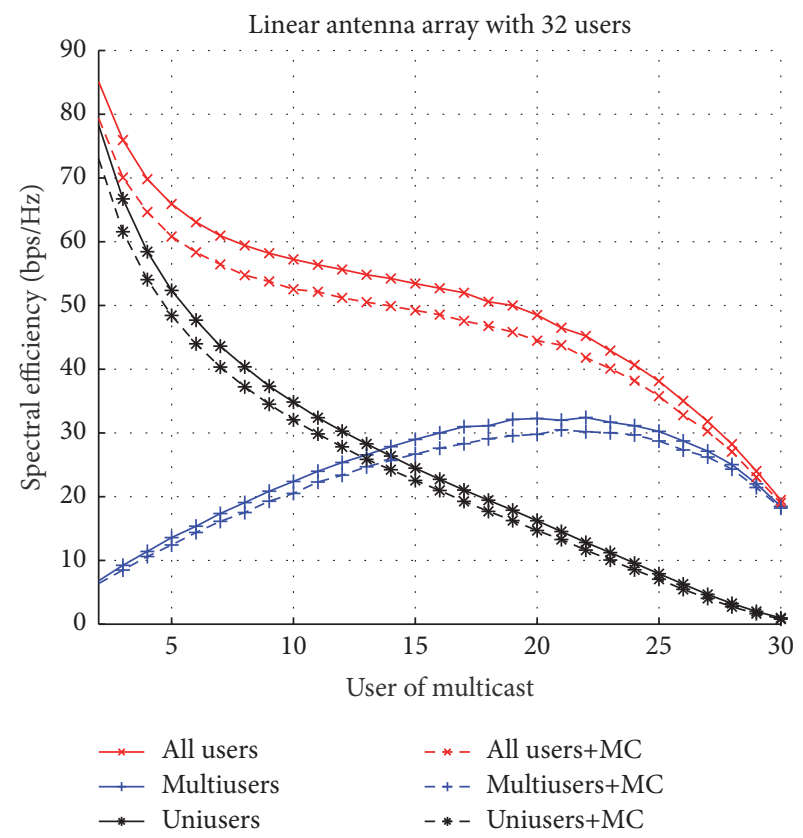

FIGURE 8: Grouping strategy analysis (linear antenna array; number of unicast UEs: 31 to 1 ; number of multicast group UEs: 1 to 31; intergroup interference cancellation: null space; $+\mathrm{MC}$ : mutual coupling; unicast precoding scheme: $\mathrm{BD}$; multicast precoding scheme: multicast precoding).

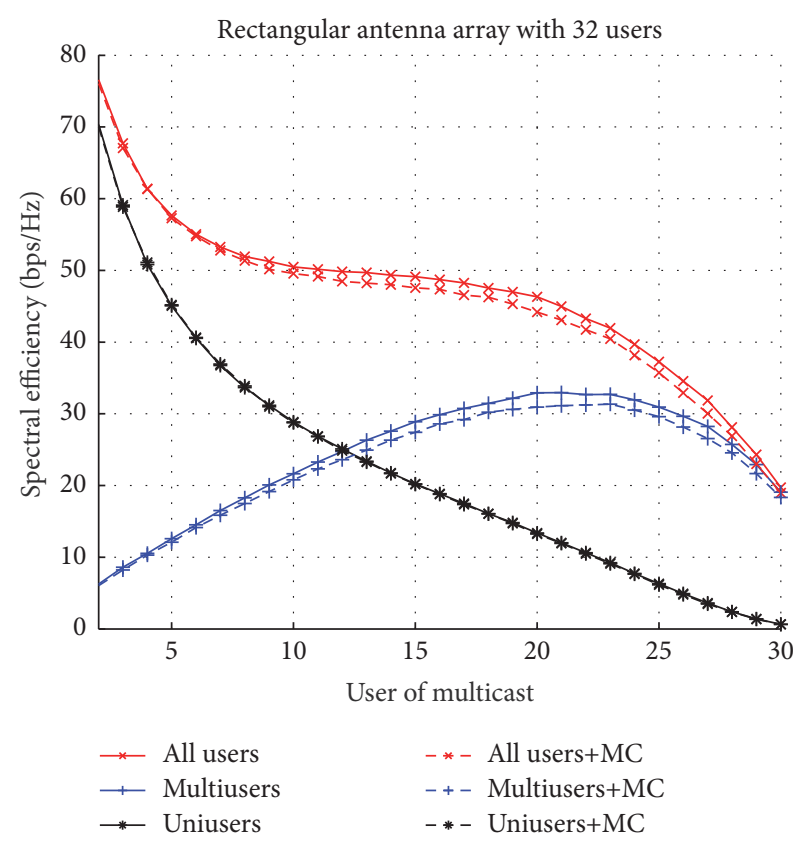

FIGURE 9: Grouping strategy analysis (rectangular antenna array, low antenna relevance; number of unicast UEs: 31 to 1; number of multicast group UEs: 1 to 31; intergroup interference cancellation: null space; $+\mathrm{MC}$ : mutual coupling; unicast precoding scheme: $\mathrm{BD}$; multicast precoding scheme: multicast precoding).

efficiency when assigning more FRs to multicast groups, which conforms with the analysis in previous subsection. This is significant for the design of antenna array in different 


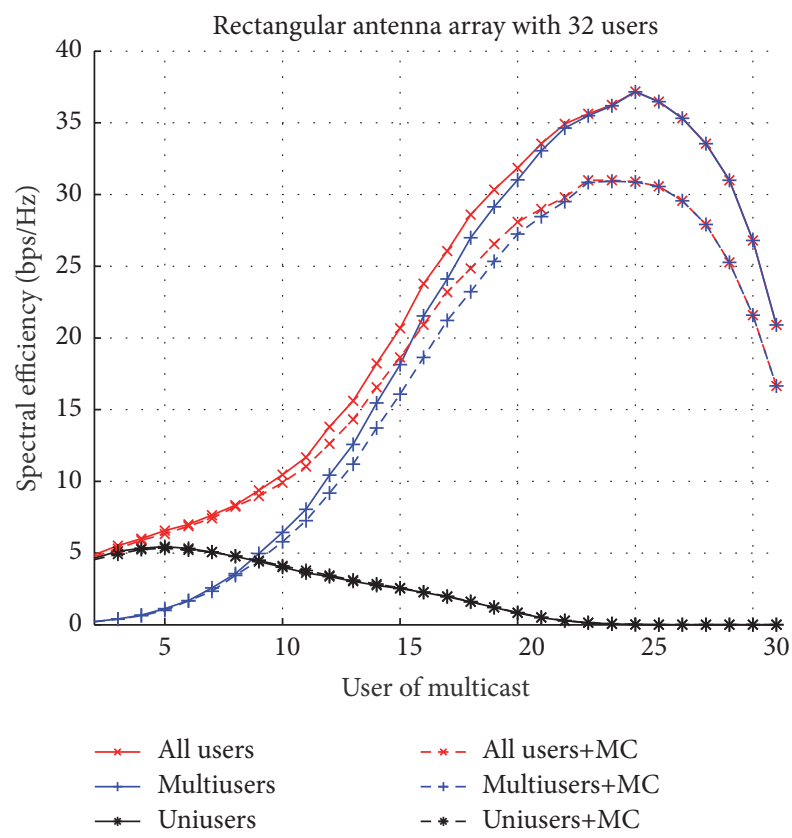

FIGURE 10: Grouping strategy analysis (rectangular antenna array, high antenna relevance; number of unicast UEs: 31 to 1; number of multicast group UEs: 1 to 31; intergroup interference cancellation: null space; $+\mathrm{MC}$ : mutual coupling; unicast precoding scheme: $\mathrm{BD}$; multicast precoding scheme: multicast precoding).

multicast service model to achieve best performance in practical wireless communication system.

\section{Conclusion}

This work investigates the wireless multicast transmission for PSN in 5G. The proposed hybrid system utilizes multicast beamforming transmission as well as the MU-MIMO linear precoding transmission. Null space method based interference cancellation is performed in the system. The simulation results indicate that under the mutual coupling channel model, the performances of different linear precoding schemes and multicast beamforming scheme degrade, while linear precoding suffers more degradation than multicast beamforming in different scenarios.

\section{Competing Interests}

The authors declare that they have no competing interests.

\section{References}

[1] A. R. McGee, M. Coutière, and M. E. Palamara, "Public safety network security considerations," Bell Labs Technical Journal, vol. 17, no. 3, pp. 79-86, 2012.

[2] A. Merwaday and I. Güvenç, "UAV assisted heterogeneous networks for public safety communications," in Proceedings of the IEEE Wireless Communications and Networking Conference Workshops (WCNCW '15), pp. 329-334, IEEE, New Orleans, La, USA, March 2015.
[3] S. Sun, B. Rong, and Y. Qian, "Artificial frequency selective channel for covert CDD-OFDM transmission," Journal of Security and Communication Networks, vol. 8, no. 9, pp. 1707-1716, 2015.

[4] J. G. Andrews, S. Buzzi, W. Choi et al., "What will 5G be?" IEEE Journal on Selected Areas in Communications, vol. 32, no. 6, pp. 1065-1082, 2014.

[5] J. Hoydis, S. Ten Brink, and M. Debbah, "Massive MIMO in the UL/DL of cellular networks: how many antennas do we need?" IEEE Journal on Selected Areas in Communications, vol. 31, no. 2, pp. 160-171, 2013.

[6] K. Zheng, S. Ou, and X. Yin, "Massive MIMO channel models: a survey," International Journal of Antennas and Propagation, vol. 2014, Article ID 848071, 10 pages, 2014.

[7] N. D. Sidiropoulos, T. N. Davidson, and Z.-Q. Luo, “Transmit beamforming for physical-layer multicasting," IEEE Transactions on Signal Processing, vol. 54, no. 6 I, pp. 2239-2251, 2006.

[8] E. Karipidis, N. D. Sidiropoulos, and Z.-Q. Luo, "Quality of service and max-min fair transmit beamforming to multiple cochannel multicast groups," IEEE Transactions on Signal Processing, vol. 56, no. 3, pp. 1268-1279, 2008.

[9] E. Matskani, N. D. Sidiropoulos, Z.-Q. Luo, and L. Tassiulas, "Efficient batch and adaptive approximation algorithms for joint multicast beamforming and admission control," IEEE Transactions on Signal Processing, vol. 57, no. 12, pp. 4882-4894, 2009.

[10] Z. Xiang, M. Tao, and X. Wang, "Massive MIMO multicasting in noncooperative cellular networks," IEEE Journal on Selected Areas in Communications, vol. 32, no. 6, pp. 1180-1193, 2014.

[11] H. Zhou and M. Tao, "Joint multicast beamforming and user grouping in massive MIMO systems," in Proceedings of the IEEE International Conference on Communications (ICC '15), pp. 1770-1775, London, UK, June 2015.

[12] S. Sun, L. Gong, B. Rong, and K. Lu, "An intelligent SDN framework for 5G heterogeneous networks," IEEE Communications Magazine, vol. 53, no. 11, pp. 142-147, 2015.

[13] N. Chen, B. Rong, A. Mouaki, and W. Li, "Self-organizing scheme based on NFV and SDN architecture for future heterogeneous networks," Mobile Networks and Applications, vol. 20, no. 4, pp. 466-472, 2015.

[14] C. Masouros, M. Sellathurai, and T. Ratnarajah, "Large-scale MIMO transmitters in fixed physical spaces: the effect of transmit correlation and mutual coupling," IEEE Transactions on Communications, vol. 61, no. 7, pp. 2794-2804, 2013.

[15] B. Clerckx, C. Craeye, D. Vanhoenacker-Janvier, and C. Oestges, "Impact of antenna coupling on $2 \times 2$ MIMO communications," IEEE Transactions on Vehicular Technology, vol. 56, no. 3, pp. 1009-1018, 2007.

[16] S. Sun, Y. Ju, and Y. Yamao, "Overlay cognitive radio OFDM system for $4 \mathrm{G}$ cellular networks," IEEE Wireless Communications, vol. 20, no. 2, pp. 68-73, 2013.

[17] F. Zhang, S. Sun, B. Rong, F. Yu, and K. Lu, "A novel massive MIMO precoding scheme for next generation heterogeneous networks," in Proceedings of the IEEE Global Communications Conference (GLOBECOM '15), San Diego, Calif, USA, 2015.

[18] E. Pakdeejit, Linear precoding performance of massive $M U$ MIMO downlink system[M.S. thesis], The Institute of Technology at Linköping University, Linköping, Sweden, 2013. 

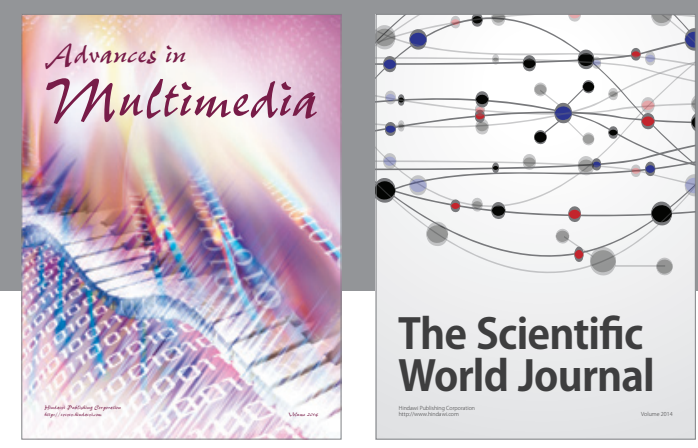

The Scientific World Journal
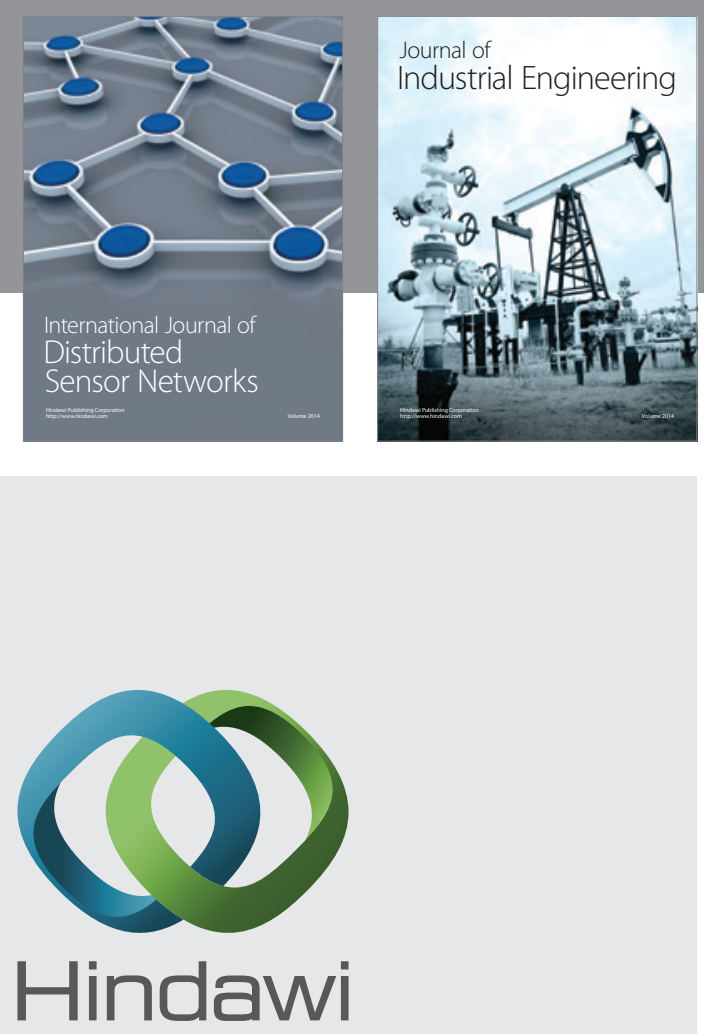

Submit your manuscripts at

http://www.hindawi.com

\section{Computer Networks} and Communications
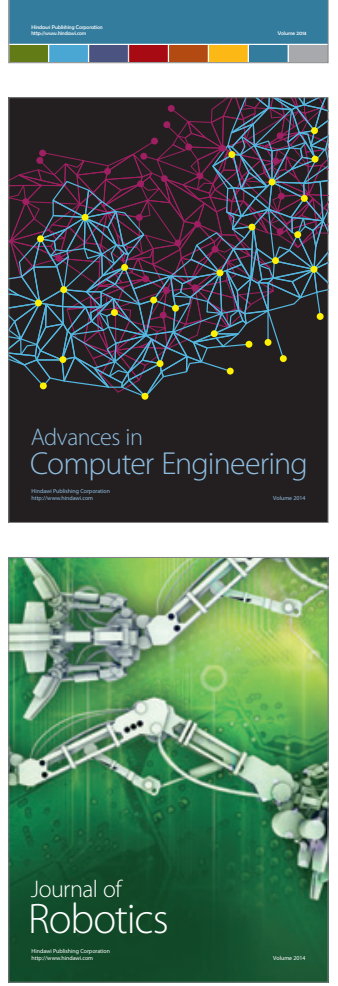
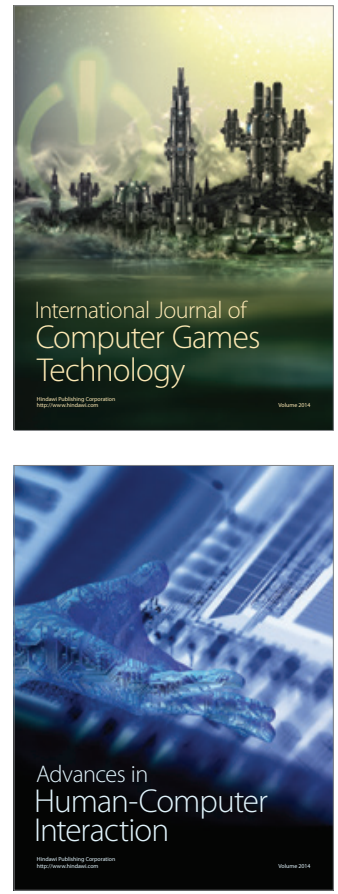
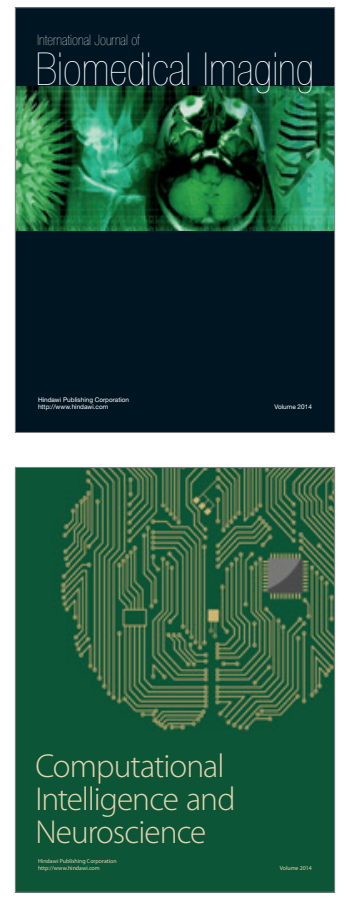
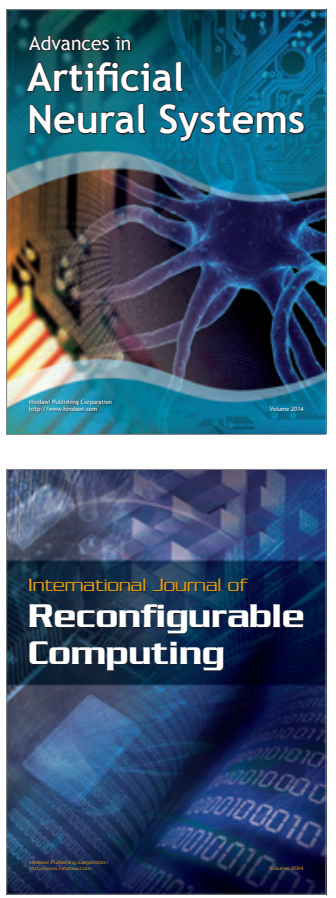
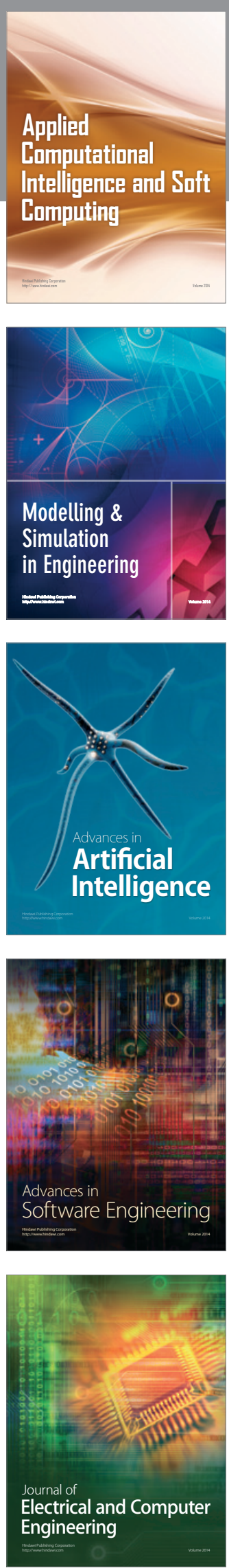